\title{
INFLUENCE OF MODIFIED CATIONIC STARCH IN A MIXED POLY(VINYL ALCOHOL)/CATIONIC STARCH SOLUTION ON THE ELECTROSPINNING PROCESS AND WEB STRUCTURE
}

\author{
Jurgita Šateikè, Rimvydas Milašius \\ Kaunas University of Technology, Faculty of Mechanical Engineering and Design, Studentu 56, LT-51424, Kaunas, Lithuania \\ E-mail: rimvydas.milasius@ktu.It
}

\begin{abstract}
:
Nanofibers were electrospun from bicomponent poly(vinyl alcohol) (PVA) and modified cationic starch (CS) mixed solution PVA/CS with different mass ratios (75/25, 50/50 and 35/65) at a total concentration of 12 wt\% for all polymer compositions. For comparison, pure PVA solution was used. Electrospinning technique Nanospider (Elmarco, Czech Republic) with a rotating electrode with tines was used to obtain nanofibrous web. The influence of prepared polymer solution compositions on the structure and morphology of nanofibers and webs were investigated. Analyzing the structure and morphology of the formed nanofiber webs, it was noticed that the fineness nanofibers were formed from the PVA/CS solution with a mass ratio of 50/50. This ratio of solution also lets us to obtain the nanofibrous web with less sticked nanofibers on spunbond. The increase in the CS ratio by more than 50/50 had a negative influence on the diameter of nanofibers and the structure of nanofibrous web.
\end{abstract}

\section{Keywords:}

electrospinning, poly(vinyl alcohol), cationic starch.

\section{Introduction}

Electrospinning technique is a versatile and efficient method to produce continuous fibers with diameters ranging from a few micrometers to a few nanometers. Various methods have been used to produce polymer nanofibers, such as selfassembly and phase separation drawing, but electrospinning is the most attractive technique due to its flexibility, simplicity, and cost-effectiveness [1-3]. This technique can be easily employed in the laboratory and can also be used for industrial process. Electrospinning has been used in many synthetic and natural polymers. Many research studies have focused on the fabrication of natural polymers using the electrospinning technique. Natural polymers are widely used in biomedical applications such as tissue engineering, medical implants, wound dressing, biosensors and drug delivery systems [47]. Electrospinning of nanofibers based on natural polymers has better possibilities for utilization of bio-based materials. From all the natural polymers, polysaccharides (starch, cellulose, alginate, chitin and chitosan) are mostly used in biomedical applications due to their unique properties, such as biodegradability, biocompatibility, nontoxicity, that promote cell growth [8-10].

Among all the polysaccharides, starch is the second abundant plant-based polymer after cellulose. Starch structure consists of two types of molecules: amylose and amylopectin. Amylose is amorphous whereas amylopectin is crystalline, and therefore starch is about $70 \%$ amorphous and $30 \%$ crystalline. Starch granules absorb the water or liquid, this causes liquid to thicken and increase in viscosity of the solution [8].
Using the electrospinning technique to form nanofibrous webs from biopolymers is quite easy, what we cannot say about nanofibers formed from pure starch. It is difficult to produce starch polymer in fibrous form due to its poor processability, water resistibility, low strength and thermal instability. There are some groups of researchers who investigated the electrospinnability of starch without inclusion of other biopolymers. It was reported that it is possible to achieve pure starch microfibers by using electrospinning technique modified with coagulation bath, which is called wet spinning technique. The solution used in this technique was obtained by heating Gelose 80 (amylose content $80 \%$ ) in dimethyl sulfoxide (DMSO) while the gelatinization of starch takes place. Also, pure ethanol was used as a solvent in the coagulation bath to achieve highly amorphous electrospun fibrous web $[11,12]$.

It was reported that using the same wet spinning technique they tried to produce microfibers from potato starch. But, the results showed that it was not possible to change the chemical nature of starch even after treating with DMSO [13].

Furthermore, some researchers investigated electrospinnability of pure starch using bioactive agents (palmitic acid [PA], ascorbyl palmitate $[\mathrm{AP}]$ and cetyl trimethyl ammonium bromide [CTAB]) during the solution preparation. The results showed that CTAB was dissolved in a coagulation bath containing ethanol in which gelatinized starch was electrospun. Meanwhile, bioactive agents PA and AP showed poor solubility in ethanol [8].

Among all the abovementioned techniques, it can be concluded that electrospinning of starch is complicated due to its chemical nature. To improve electrospinnability, the starch 
can be modified or blended with synthetic polymers to provide the structural integrity and hydrophilicity to the mat. Poly(vinyl alcohol) (PVA) polymer has been used for producing nanofibers via the electrospinning technique due to its good properties such as chemical and thermal stability, hydrophilicity and nontoxicity $[9,10,14]$. Biodegradable natural polysaccharides can be easily modified to cationic derivatives of starch $[9,10]$.

The main aim of this work was to form nanofibers via the electrospinning technique with PVA and cationically modified starch CS spinning solution, to find out which amount of CS is preferable into spinning solution, to analyze CS influence on electrospinning process and the structure of nanofibrous webs and to compare the results with the electrospun nanofibers from pure PVA solution.

\section{Experimental}

PVA solution was prepared by dissolving PVA powder (Roth, Germany; $M=72,000 \mathrm{~g} / \mathrm{mol}$ ) in distilled water at $85^{\circ} \mathrm{C}$ under constant stirring for $2 \mathrm{~h}$ with a final solid concentration of 12 wt $\%$. Several types of polymer solutions were prepared with a total concentration of $12 \mathrm{wt} \%$ for the electrospinning process. Electrospinning solutions of PVA/CS with different mass weight ratios of $75 / 25,50 / 50$ and $35 / 65$ were prepared to find out which amount of CS is preferable for the electrospinning process. Pure PVA solution was prepared to compare the results of the electrospinning process with those of the mixed PVA/CS solutions at different mass ratios.

Nanofibrous webs were formed by Nanospider (Elmarco, Czech Republic) electrospinning equipment. On this equipment, the rotating electrode with tines is covered by a film of polymer solution. Increase in the applied voltage between the electrodes leads to the formation of Taylor cones on the electrode. Only when the electrostatic force overcomes the surface tension of the polymer solution, a jet of polymer solution is ejected from the Taylor cone. The jet moves toward the upper electrode and sets down on the substratum material - spunbond from polypropylene filaments (thickness of spunbond, $0.125 \pm 0.015 \mathrm{~mm}$; surface mass, $21.1 \pm 3 \mathrm{~g} / \mathrm{m}^{2}$ ). Meanwhile, the nanofiber becomes thinner, the solvent evaporates and then the fibers solidify.

During all the experiments, the distance between the electrodes was $13 \mathrm{~cm}$, the electrical potential applied varied between 35 and $70 \mathrm{kV}$, the temperature of the electrospinning environment was $t=20 \pm 2^{\circ} \mathrm{C}$, and the relative air humidity was $\varphi=43 \pm 2 \%$.

The structure of nanofibrous web was determined using a scanning electron microscope (SEM) (Quanta 200 FEG; FEI, The Netherlands). The diameter of nanofibers was measured using image analysis system LUCIA 5.0 from every SEM image (five SEM images for each kind of electrospun web were used).

\section{Results and discussion}

As mentioned earlier, it is currently very complicated to form nanofibers using the electrospinning technique from pure starch or starch derivatives. For this reason, nanofibers from electrospinning solutions in which one of the components was modified cationic starch (CS) were formed in this work.

Our previous work described the attempt to form nanofibers with a PVA/CS (mass ratio 75/25) solution at different concentrations $(8,10$, and $12 w t \%)$ using two types of rotating electrodes: plain cylindrical and electrode with tines [10]. The results showed that by using electrode with tines, the diameter of nanofibers was significantly decreased and nanofibers with higher density were formed. Furthermore, the concentration of the spinning solution using a rotating electrode with tines did not affect the diameter of the nanofibers. Meanwhile, the concentration of the electrospinning solution influenced the diameter using a plain cylindrical electrode. For this reason, in this experiment nanofibers were electrospun from concentration of $12 \mathrm{wt} \% \mathrm{PVA} /$ CS polymer solution with different mass ratios of $75 / 25,50 / 50$ and $35 / 65$ using a rotating drum electrode with tines. The main objective of this work was to analyze what content of CS into spinning solution is preferable to form nanofibers and compare the results with the same concentration of pure PVA polymer solution. At the first stage of investigation, the minimal range of applied voltage for the electrospinning process was determined. It was found out that the electrospinning process using pure PVA solution and PVA/CS solution with mass ratio 75/25 starts at the level of $35 \mathrm{~V}$, while with higher ratio of CS the higher applied voltage is needed - process started only at $45 \mathrm{~V}$. It means that starch has negative influence on the electrospinning process as for solution with higher CS ratio a higher applied voltage is needed. The results were confirmed by other researchers that also noted the difficulties of electrospinning process in solution with starch $[8,13]$. The maximum level of applied voltage of the electrospinning process was determined at the level of 65$70 \mathrm{~V}$. It is necessary to note that pure PVA and PVA/CS 75/25 solutions can be electrospun even at $70 \mathrm{~V}$, whereas solutions with higher CS ratio only till $65 \mathrm{~V}$. In the case of higher applied voltage the discharge appears. The SEM images of the formed nanofibrous webs are presented in Figure 1.

As shown in Figure 1, in the case of PVA/CS ratio 75/25 less dense structure of web was obtained. This can be explained by the fact that in this case applied voltage was $70 \mathrm{~V}$, whereas for other variants of blended solutions it was lower, i.e. $65 \mathrm{~V}$. The average values of diameter of electrospun nanofibers for all webs are presented in Table 1.

At the next step of investigation, the distributions of all nanofibers diameters were calculated for further analysis (Figure 2).

As shown in Figure 2, the diameter distributions of nanofibers formed from pure PVA and PVA/CS with lower CS mass ratio $(75 / 25)$ is high - even in the level of $750-900 \mathrm{~nm}$. Such thick fibers were formed as the nanofibers stick together on the surface of spunbond. Herewith, the modal value of diameters also depends on the CS ratio of solution and this dependency is very similar to the dependency of average values of nanofibers - increasing CS ratio modal value of nanofibers decreases. While in the case of PVA/CS 65/35 ratio, the modal value increased (Figure 2 and Table 1). 


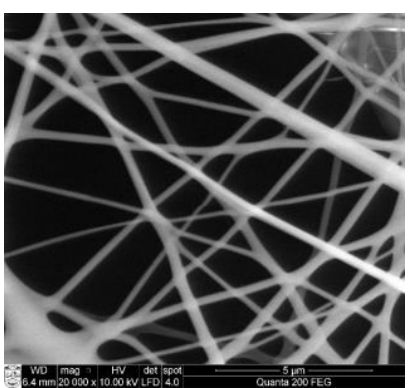

a)

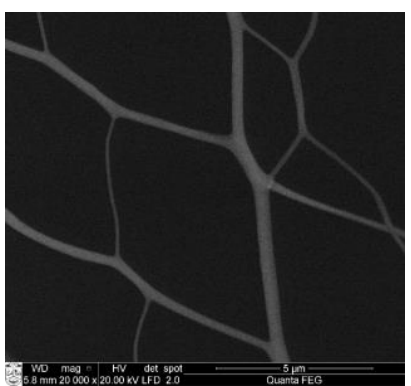

b)

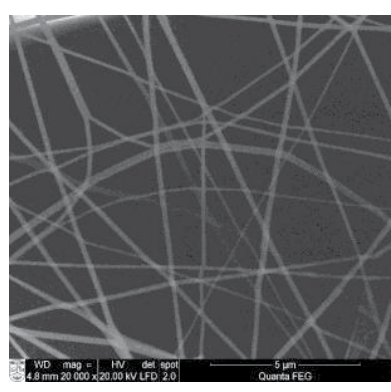

c)



d)

Figure 1. Scanning electron microscope images of nanofibrous webs: (a) pure poly(vinyl alcohol) (PVA); (b) PVA/cationic starch (CS) 75/25 mass ratio; (c) PVA/CS 50/50 mass ratio; and (d) PVA/CS 35/65 mass ratio.
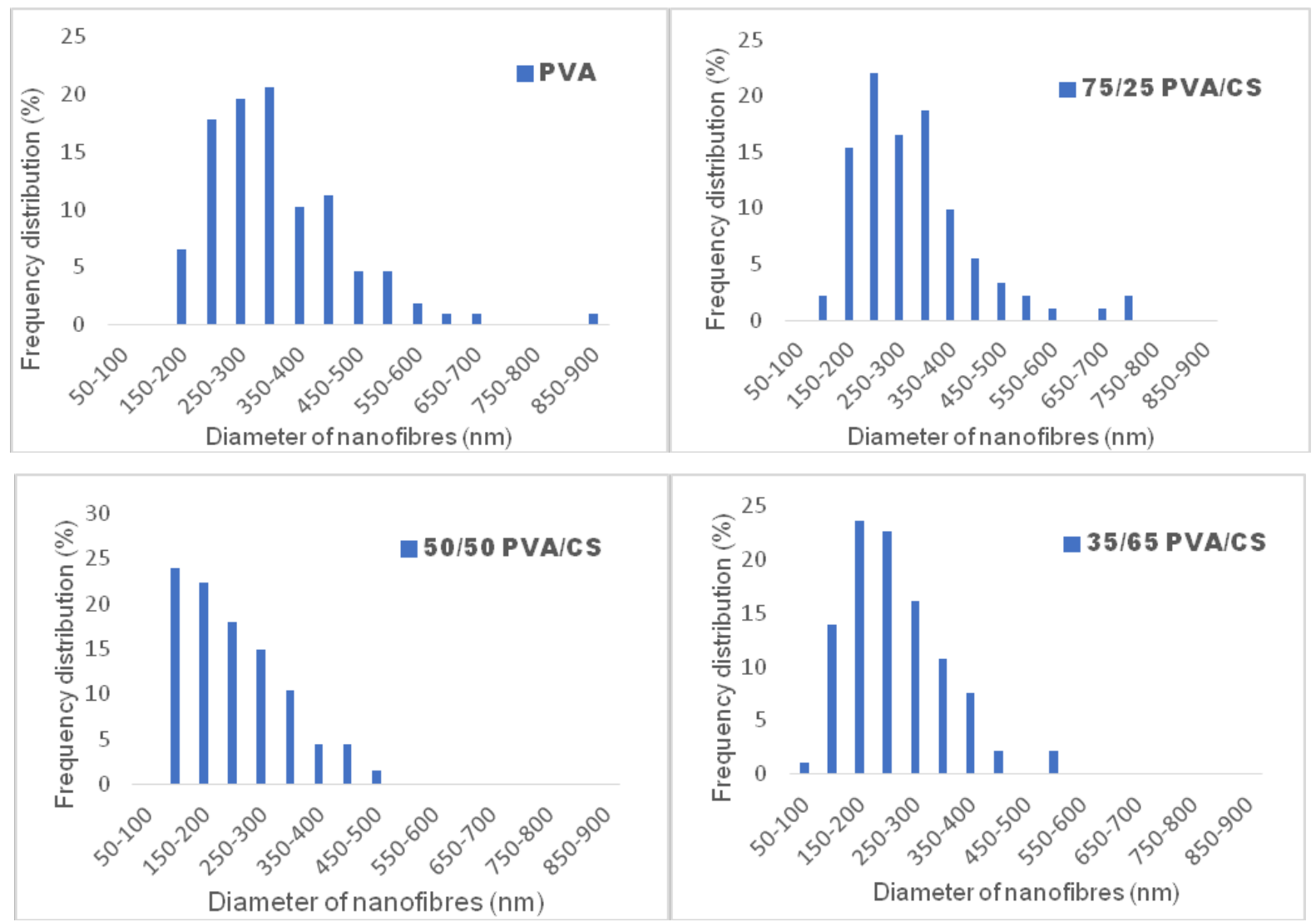

Figure 2. Diameter distribution of nanofibers formed from pure poly(vinyl alcohol) (PVA) and PVA/cationic starch (CS) solutions

Table 1. Average and modal values of the diameter of nanofibrous webs

\begin{tabular}{|c|c|c|}
\hline Solution & Average value, $\mathbf{n m}$ & Modal value, $\mathbf{n m}$ \\
\hline Pure PVA & 336 & 325 \\
\hline PVA/CS 75/25 & 301 & 225 \\
\hline PVA/CS 50/50 & 226 & 125 \\
\hline PVA/CS 35/65 & 238 & 175 \\
\hline
\end{tabular}

CS, cationic starch; PVA, poly(vinyl alcohol). 
The analysis of distributions showed the positive CS influence on the diameter of nanofibers - average and modal values of diameters are lower. Meanwhile, in the case of highest amount of CS (ratio 65/35) the diameter of nanofibers increased and the sticked nanofibers appear again. The obtained results can be explained by properties of components. It is known that the viscosity of solution with CS is higher than pure PVA $[8,10]$. Due to higher viscosity of the blended solution, the process of solidification became more intensive compared with pure PVA solution or that with low quantity of CS. This affects the sticking of nanofibers. In the case of high ratio of CS in solution (65/35), the other factor starts to play a significant role - starch creates difficulties in the formation of nanofibers via the electrospinning process. The SEM image (Figure 1d) confirms this hypothesis - structure of web was formed from sophisticated constructions of fibers, not from the single fiber. So, the optimal ratio of CS in PVA/CS solution would be $50 / 50$.

\section{Conclusion}

The quantity of starch in solution affects the electrospinning process - at higher ratio of CS the higher applied voltage is needed for electrospinning process. Although the applied voltage of $70 \mathrm{~V}$, which is acceptable for pure PVA and PVA/CS $75 / 25$ ratio solutions, is too high for web formation using PVA/ CS 50/50 and 35/65 ratio solutions, the discharges appear. The amount of CS in the electrospinning solution had positive influence on the diameter of nanofibers. By increasing the CS ratio till 50/50 the average and modal values of nanofiber diameter decreased. Also, the web is formed with less sticked fibers. It can be stated that the PVA/CS ratio $50 / 50$ is the optimal ratio for the fineness nanofibrous web with starch formation. For more precise optimal ratio determination, a further investigation using the PVA/CS ratio from $70 / 30$ to $40 / 60$ is needed.

\section{References}

[1] Bhardwaj, N., Kundu, S. C. (2010). Electrospinning: a fascinating fiber fabrication technique. Biotechnology Advances, 28, 325-347.

[2] Huang, Z. M., Zhang, Y. Z., Kotaki, M., Ramakrishna, S. (2003). A review on polymer nanofibres by electrospinning and their applications in nanocomposites. Composites Science and Technology, 63(15), 2223-2253.

[3] Kleivaite, V., Milašius, R. (2018). Electrospinning - 100 Years of investigations and still open questions of web structure estimation. AUTEX Research Journal, 4(18), 398-404.
[4] Stijnman, A. C., Bodnar, I., Tromp, R. H. (2011). Electrospinning of food-grade polysaccharides. Food Hydrocolloids, 25, 1393-1398.

[5] Hualin, W., Wenjuan, W., Suwei, J., Shoatong, J., Linfeng, Z., Qin, Z. (2011). Poly(vinyl alcohol)/oxidized starch fibres via electrospinning technique: fabrication and characterization. Iranian Polymer Journal, 20(7), 551-558.

[6] Sutka, A., Kukle, S., Gravitis, J., Milašius, R., Malašauskiene J. (2013). Nanofibre electrospinning poly(vinyl alcohol) and cellulose composite mats obtained by use of a cylindrical electrode. Advances in Materials Science and Engineering. 2013 (2013), DOI:10.1155/2013/932636.

[7] Goktepe, F., Mulayim, B. B. (2018). Long path towards to success in electrospun nanofiber yarn production since 1930's: a critical review. Autex Research Journal, 2(18), 87-109.

[8] Lee, K. Y., Jeong, L., kang, Y. O., Lee, S. J., Park, W. H. (2009). Electrospinning of polysaccharides for regenerative medicine. Advanced Drug Delivery Reviews, 51, 10201032.

[9] Adomavičiūtè, E., Milašius, R., Žemaitaitis, A., Bendoraitienè, J., Leskovšek, M., Demšar, A. (2009). Methods of forming nanofibres from bicomponent PVA cationic starch solution. Fibres \& Textiles in Eastern Europe, 3(74), 29-33.

[10] Šukytè, J., Adomavičiūtè, E., Milašius, R., Bendoraitienè, J., Danilovas, P. P. (2012). Formation of Poly(Vinyl Alcohol)/ cationic starch blend nanofibres via the electrospining technique: the influence of different factors. Fibres \& Textiles in Eastern Europe, 3(92), 16-20.

[11] Thillaipandian, H., Vankateshwarapurum, R. G. D. (2017). Comprehensive review on electrospinning of starch for biomedical applications, International Journal of Biological Macromolecules, 106, 712-718.

[12] Kong, L., Zeigler, G. R. (2014). Fabrication of pure starch fibers by electrospinning, Food Hydrocolloids, 36, 20-25.

[13] Cardenas, W., Gomez-Pachon, E. Y. G., Munaz-Graziano, R. (2016). Preparation of potato starch microfibres obtained by electro wet spinning, Materials Science and Engineering, 138.

[14] Talmoudi, H., Khenoussi, N., Adolphe, D., Said, A. H., Schacher, L. (2018). An in situ crystal growth of metal organic frameworks-5 on electrospun PVA nanofibers. AUTEX Research Journal, 3(18), 308-313. 